\title{
Infrared physics in inflation and primordial perturbations
}

\section{Yuko Urakawa*}

Institute for Advanced Research, Nagoya University

E-mail: urakawa.yuko@h.mbox.nagoya-u.ac.jp

This talk is based on our works [2, 3] and our review article [1] about loop corrections of the primordial perturbations generated during inflation. A naive perturbation theory predicts that loop corrections generated during inflation suffer from various infrared (IR) divergences. We discuss the origin of the IR divergences and explore the regularity conditions, which will restrict the possible initial states of inflationary universe.

References

[1] T. Tanaka and Y. Urakawa, Class. Quant. Grav. 30, 233001 (2013) [arXiv:1306.4461 [hepth]].

[2] T. Tanaka and Y. Urakawa, PTEP 2013, no. 6, 063E02 (2013) [arXiv:1301.3088 [hep-th]].

[3] T. Tanaka and Y. Urakawa, arXiv:1402.2076 [hep-th], accepted in PTEP.

Frontiers of Fundamental Physics 14 - FFP14,

15-18 July 2014

Aix Marseille University (AMU) Saint-Charles Campus, Marseille

\footnotetext{
${ }^{*}$ Speaker.
} 